\title{
NUEVOS RESTOS DE UN ODONTOCETI FÓSIL (MAMMALIA: CETACEA, PHYSETEROIDEA) PARA EL MIOCENO TARDÍO DE PANAMÁ, AMÉRICA CENTRAL
}

\author{
NEW FOSSIL REMAINS OF AN ODONTOCETI (MAMMALIA: CETACEA, \\ PHYSETEROIDEA) FROM THE LATE MIOCENE OF PANAMA, CENTRAL AMERICA
}

\author{
Dioselina I. Vigil ${ }^{1} \& 2$ \& César A. Laurito ${ }^{3 \& 4 *}$ \\ ${ }^{1}$ Escuela de Biología de la Universidad de Panamá \\ ${ }^{2}$ Instituto de Investigaciones Tropicales del Smithsonian (STRI). \\ Apartado postal: 0818-00423, Panamá, Rep. de Panamá \\ ${ }^{3}$ Instituto Nacional de Aprendizaje (INA) \\ ${ }^{4}$ Investigador Asociado-Departamento de Historia Natural, \\ Museo Nacional de Costa Rica \\ *Autor para contacto: cesarlaurito@ice.co.cr
}

(Recibido: 20/01/2014; aceptado: 10/05/2014)

\begin{abstract}
A fragment of a crown and an isolated lower commissural tooth of a sperm whale of the Family Physetheridae are described. The dental remains were recovered from the upper bathyal sediments of the Chagres Formation (late Miocene), with a strong Pacific affinity in the locality of the Piña Town in the Caribbean littoral of Panama. Keywords: Mammalia, Cetacea, Physetheridae, Late Miocene, Chagres Formation, Panama.

RESUMEN: Un fragmento de corona y un diente aislado, inferior y comisural de una ballena de esperma de la Familia Physeteridae son descritos. Los restos dentales fueron colectados en sedimentos batiales superiores de la Formación Chagres (Mioceno Tardío) con fuerte afinidad del Pacifico en la localidad de el poblado de Piña en el litoral caribeño de Panamá.

Palabras clave: Mammalia, Cetacea, Physetheridae, Mioceno Tardío, Formación Chagres, Panamá.
\end{abstract}




\section{INTRODUCCIÓN}

El registro fósil de mamíferos marinos para América Central es en general escaso e infrecuente; por muchos años el único registro conocido se debió a Reshetov, 1981, quién menciona el cetáceo de la Formación El Salto en el Suroeste de Nicaragua, conformada por sedimentos marino someros tanto clásticos como carbonaticos y de edad Plioceno tardío (sensu Kolb \& Schmidt, 1991). Este hallazgo fue estudiado por Lucas et al., 2009, quienes lo determinaron como un Balaenopteridae indeterminado.

En años recientes se han dado a conocer nuevos hallazgos de Odontoceti procedentes de dos localidades de Costa Rica, donde se han descrito restos de Squalodon un Squalodontidae del Mioceno Medio de la Formación Río Banano (Laurito et al., 2011) y los géneros Eurhinodelphis, Goniodelphis, Hadrodelphis y Orycterocetus de las correspondientes familias Eurhinodelphinidae, Iniidae, Kentriodontidae y Physeteridae, todos procedentes del Mioceno Superior de la Formación Curré (Valerio \& Laurito, 2011). Por su parte en Panamá, Uhen et al. (2010) dan a conocer el registro de varios restos post-craneales de Odontoceti indeterminado procedentes del Serravaliano de la Formación Gatún y de un Balaenopteroidea? indeterminado del Mesiniano de la Formación Tobabe; además, describen de restos de Sirenia, uno indeterminado de edad Aquitaniano y un Dugongidae de edad Burdigaliano de la Formación Culebra.

El objetivo de la presente nota es dar a conocer el material dental de un Odontoceti, recuperado de la plataforma de abrasión marina de playa Piña, en las cercanías del pueblo del mismo nombre, en el Corregimiento de Piña, Provincia de Colón, República de Panamá; en las coordenadas $9^{\circ} 16^{\prime} 52.58^{\prime \prime} \mathrm{N}$ y $80^{\circ}$ 2' 52.99 " W, hoja 4143 I Palmas Bellas, Instituto Geográfico Nacional de Panamá, Tommy Guardia (Fig. 1).

\section{PALEONTOLOGÍA SISTEMÁTICA}

Cetacea Brisson, 1762

Odontoceti Flower, 1867

Physeteroidea (Gray, 1821) Gill, 1972

Physeteridae Gray, 1821

Género y especie indet.

Material: el DVP-9912-5 (Fig. 2, 1a-c) corresponde a un molar inferior próximo a la comisura y de posición incierta; el DVP-9912-7 (Fig. 2, 2) corresponde a un fragmento de corona aislado. Ambos están depositados en el Museo de Vertebrados de la Universidad de Panamá, bajo el número de catálogo MVUP-1722.

Descripción: el DVP-9912-5 es un molar homodonto, transversalmente aplanado, muy robusto con corona cónica relativamente pequeña, volcada ligeramente en sentido comisural y esmalte ornamentado con estrías longitudinales. La raíz es conspicuamente inflada en sus dos tercios superiores con sus facetas transversales aplanadas y el tercio inferior, aunque roto, es notablemente angosto y cilíndrico. La porción superior o expuesta de la raíz (sensu Bianucci \& Landini, 2006) presenta una amplia región gingival donde se observan profundas facetas de oclusión.

La altura total del diente es $53,9 \mathrm{~mm}$ y de la corona 14,3 $\mathrm{mm}$ con un ancho basal de 11,7 mm; la raíz presenta un ancho máximo de $27,8 \mathrm{~mm}$ y su porción inferior un ancho máximo de 19,4 mm y ancho mínimo de 19,1 mm.

El DVP-9912-7 corresponde a un fragmento de corona cónica con estrías longitudinales, su altura máxima es de $16,9 \mathrm{~mm}$ y su ancho basal máximo de 11,9 mm.

Edad: los restos proceden de los acantilados costeros en los alrededores de la desembocadura del río Piña que de acuerdo a Collins et al., 1996, corresponden a la Formación Chagres y están constituidos por bancos de areniscas gruesas a 


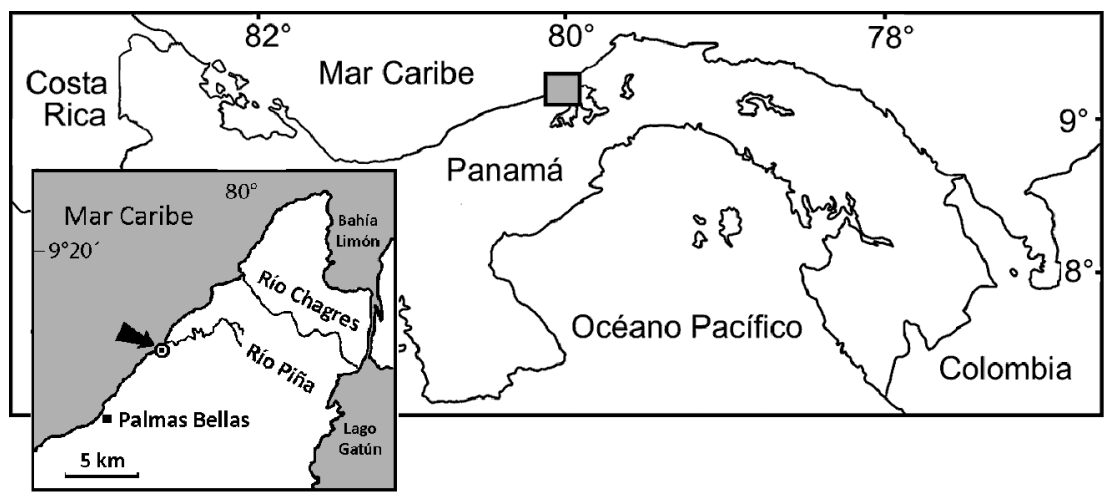

Fig. 1: ubicación del sitio del hallazgo en las proximidades de la desembocadura del río Piña, Provincia de Colón, República de Panamá.

medias, limos arcillosos y areniscas coquinoides (sensu Escalante, 1990), depositados en un ambiente batial superior (200 a $500 \mathrm{~m}$ ) con faunas plantónicas de marcada afinidad Pacífico NorteEcuatorial y de edad Mioceno Superior (6 Ma).

Discusión y conclusiones: el molar descrito, presenta características propias de las ballenas de esperma como lo son el gran tamaño del diente, la pequeña corona estriada y la presencia de las profundas facetas de oclusión en la porción gingival de la raíz que denotan una muy corta distancia entre los dientes (sensu Lambert et al., 2008). Todos estos caracteres más la retención del esmalte en la corona sugiere que se trata de un Physeteroidea basal.

La morfología general del diente recuerda a los géneros Zygophyseter Bianucci \& Landini, 2006 del Mioceno Superior de Italia y "Scaldicetus" común en yacimientos del Mioceno de Australia, Europa, Norte y Suramérica y (Fitzgerald, 2004; Whitmore \& Kaltembach, 2008), pero se diferencia de estos por tener una corona más alineada con el eje de la raíz y por ser esta mucho más inflada.

Todas las características arriba descritas, permiten concluir que el diente de Panamá es similar y muy cercano al género Acrophyseter Lambert, Bianucci \& de Muizon, 2008 presente en el Mioceno tardío de Perú, pero por tratarse de material aislado y fragmentario no es posible en este caso asignarle un género con certeza por su similitud con otros géneros de la familia.

Por último, cabe indicar que aunque la corona no presenta desgaste evidente, la preservación de la cavidad pulpar sugiere que se trata de un individuo maduro.

\section{REFERENCIAS BIBLIOGRÁFICAS}

BIANUCCI, G. \& LANDINI, W., 2006: Killer sperm whale: a new basal physeteroid (Mammalia, Cetacea) from the Late Miocene of Italy.- Zoological J. Linnean Soc. 148: 103-131.

COLLINS, L.S., COATES, A.G., BERGGREN, W.A., AUBRY, M-P., ZHANG, J., 1996: The Late Miocene Panama isthmian strait.Geology, 24: 687-690.

ESCALANTE, G., 1990: Geology of Southern Central America and Western Colombia.En: Dengo, G. \& Case, J.E. (eds): The Caribbean Region.-The Geology of North America, H: 201-230. 


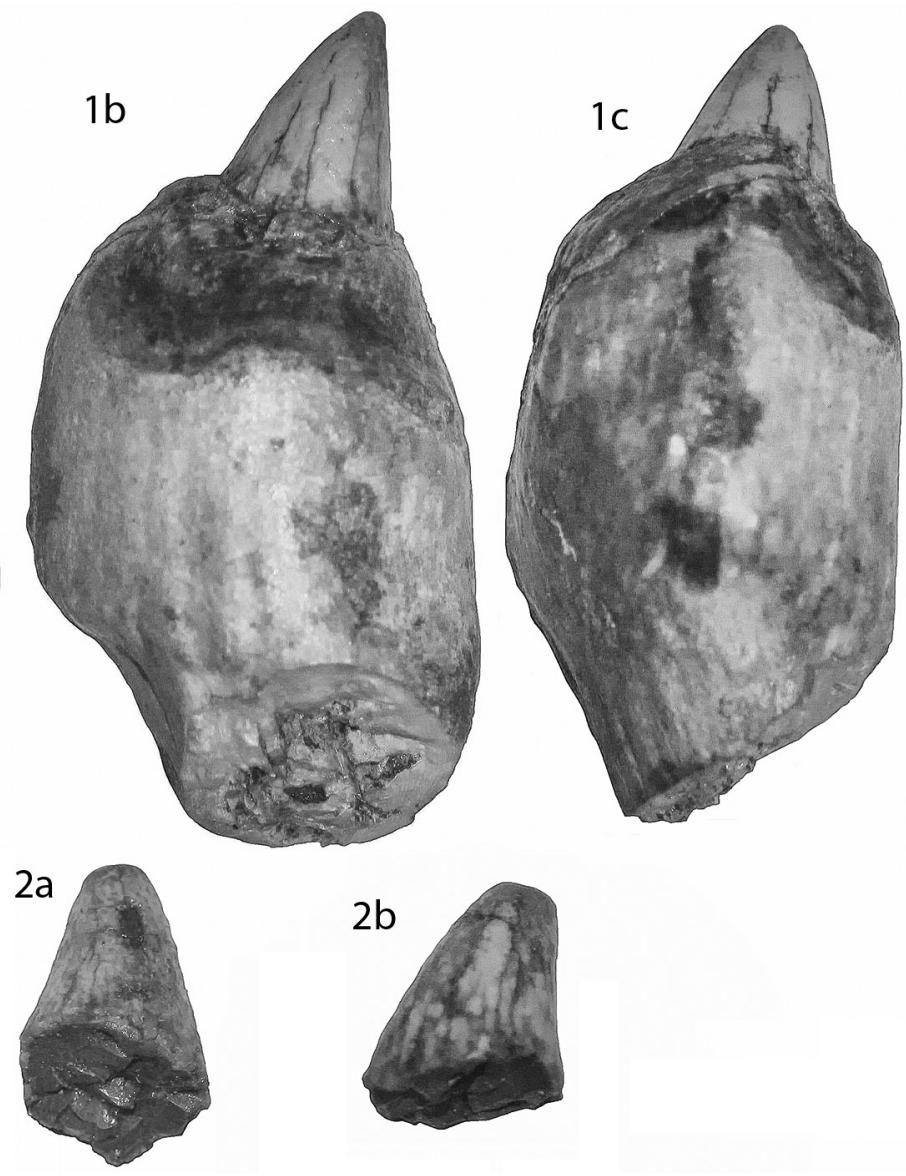

Fig. 2: Physeteridae indet., 1. DVP-9912-5 molar inferior próximo a la comisura, a. en norma distal, b. oblicua y c. lateral. 2. DVP9912-7 fragmento de corona, en norma a. distal y b. lateral. Escala visual $1 \mathrm{~cm}$.

FITZGERALD, E.M.G., 2004: A review of the Tertiary fossil Cetacea (Mammalia) localities in Australia.- Memoirs of Mus. Victoria, 61(2): 183-208.

KOLB, W. \& SCMIDT, H., 1991: Depositional sequences associated with equilibrium coastline in the Neogene of Southwestern Nicaragua.- En: MACDONALD, D.I.M. (ed.): Sedimentation, tectonics and eustasy: sea-level changes at active margins.- Int. Sedimentologists Spec. Pub. 12: 259-272.
LAMBERT, O., BIANUCCI, G. \& MUIZON, C. de, 2008: A new stem-sperm whale (Cetacea, Odontoceti, Physeteroidea) from the Latest Miocene of Peru.- Comptes Rendus Paleoevol. 7: 361-369.

LAURITO, C.A., VALERIO, A.L.HERNÁNDEZ, A.C. \& OVARES, E., 2011: Primer registro de un cetáceo fósil (Mammalia, Cetacea, Odontoceti, Squalodontidae) en la Formación Río Banano, Mioceno Medio de Costa Rica, América Central.- Rev. Geol. Amér. Central, 44: 153-156. 
LUCAS, S.G, MCLEOD, S.A., BARNES, L.G., ALVARADO, G.E., GARCÍA, R. \& ESPINOZA, E., 2009: A baleen whale from the Pliocene of Nicaragua.- Rev. Geól. Amér. Central, 41: 17-24.

RESHETOV, V.J., 1982: Reporte preliminar sobre la observación de la paleontología de Nicaragua.- 13 págs.- Mus. Nac. Nicaragua, Managua.

UHEN, M.D., COATES, A.G., JARAMILLO, C.A., MONTES, C., PIMIENTO, C., RINCÓN, A., STRONG, N., VELEZJUARBE, J., 2010: Marine mammals from the Miocene of Panama.- J. South Amer. Earth Sci. 30: 167-175.
VALERIO, A.L. \& LAURITO, C.A., 2012: Cetáceos fósiles (Mammalia, Odontoceti, Eurhinodelphinoidea, Inioidea, Physeterioidea) de la Formación Curré, Mioceno Superior (Hemphilliano temprano tardío) de Costa Rica.- Rev. Geol. Amér. Central, 46: 151-160.

WHITMORE, F. C. JR. \&. KALTENBACH, J. A., 2008: Neogene Cetacea of the Lee Creek Phosphate Mine, North Carolina. - En: RAY, C.E., BOHASKA, D.J., KORETSKY, I.A., WARD, L.W. \&. BARNES L.G. (eds): Geology and paleontology of the Lee Creek Mine, North Carolina, IV.- Virginia Mus. Natural Hist. Spec.Pub. 14: 181-269. 
